\title{
Opposing Effects of Contextual Surround in Human Early Visual Cortex Revealed by Functional Magnetic Resonance Imaging with Continuously Modulated Visual Stimuli
}

\author{
Satohiro Tajima, ${ }^{1,2 \star}$ Masataka Watanabe, ${ }^{2 \star}$ Chihiro Imai, ${ }^{2}$ Kenichi Ueno, ${ }^{3}$ Takeshi Asamizuya, ${ }^{3}$ Pei Sun, ${ }^{3}$ Keiji Tanaka, ${ }^{3}$ \\ and Kang Cheng ${ }^{3}$ \\ ${ }^{1}$ Department of Complexity Science and Engineering, The University of Tokyo, Kashiwa-shi 277-8561, Japan, ${ }^{2}$ Department of Quantum Engineering and \\ Systems Science, The University of Tokyo, Tokyo 113-0033, Japan, and ${ }^{3}$ RIKEN Brain Science Institute, Wako-shi 351-0198, Japan
}

Spatial context in vision has profound effects on neural responses and perception. Recent animal studies suggest that the effect of surround on a central stimulus can dramatically change its character depending on the contrast of the center stimulus, but such a drastic change has not been demonstrated in the human visual cortex. To examine the dependency of the surround effect on the contrast of the center stimulus, we conducted an functional magnetic resonance imaging experiment by using a low or a high contrast in the center region while the surround contrast was sinusoidally modulated between the two contrasts. We found that the blood oxygen leveldependent response in human V1 corresponding to the center region was differentially modulated by the surround contrast, depending crucially on the center contrast: whereas a suppressive effect was observed in conditions in which the center contrast was high, a facilitative effect was seen in conditions where the center contrast was low.

\section{Introduction}

Spatial context in a visual stimulus has profound effects on both neural responses and perception, which is widely known as "contextual modulation.” Animal studies using single-unit recording (Blakemore and Tobin, 1972; Nelson and Frost, 1985; Knierim and van Essen, 1992; Li and Li, 1994; Kapadia et al., 1995; Sillito et al., 1995; Zipser et al., 1996; Polat et al., 1998; Sceniak et al., 1999; Kasamatsu et al., 2001) and intrinsic optical imaging (Toth et al., 1996) have shown that responses of V1 neurons to the target grating stimulus presented inside the classical receptive field can be modulated by a contextual collinear stimulus falling outside the classical receptive field. The polarity of the effect depends on the target contrast. In general, suppression occurs when the contrast of the central target is relatively high while facilitation occurs when it is low. In humans, a study using visually evoked potential has implied the existence of contrast-dependent suppression and facilitation (Polat and Norcia, 1996), but precise cortical loci responsible for such contrast-dependent responses are not identified in the study. Although several studies using noninvasive imaging approaches, such as functional magnetic resonance imaging (fMRI) (Williams et al., 2003; Zenger-Landolt and Heeger, 2003) and magnetoencephalography (Ohtani et al., 2002), have

Received Sept. 1, 2009; revised Dec. 13, 2009; accepted Jan. 14, 2010.

This work was partially supported by Ministry of Education, Culture, Sports, Science, and Technology Grants 17022015, 18020033, and 20020033 and Japan Society for the Promotion of Science Grant 20300114.

*S.T. and M.W. contributed equally to this work.

Correspondence should be addressed to either of the following: Satohiro Tajima at his present address: Nagano Station, The Japan Broadcasting Corporation, 210-2 Inaba, Nagano-City, 380-8502, Japan, E-mail: tajima. s-iu@nhk.or.jp; or Masataka Watanabe at the above address, E-mail: watanabe@bs.t.u-tokyo.ac.jp.

D01:10.1523/JNEUROSCI.4473-09.2010

Copyright $\odot 2010$ the authors $\quad 0270-6474 / 10 / 303264-07 \$ 15.00 / 0$ documented the suppressive responses as a consequence of the surround modulation in human visual cortex, there has been no evidence for the contrast-dependent switching between suppressive and facilitative responses. Overall, it is difficult to detect these weak modulatory effects, especially when the contrast of the target region is low. In the present fMRI study, we have introduced a novel stimulation design, in which the contrast of the surround stimulus was slowly and continuously modulated over the stimulation period. This approach enabled our analyses to be more sensitive to the modulatory responses embedded in the blood oxygen level-dependent (BOLD) signal that typically contains other unrelated response features as well. Our results demonstrated clearly that, under otherwise identical experimental conditions, the polarity of the contextual effect changes depending solely on the contrast of target stimulus.

\section{Materials and Methods}

Subjects. Three healthy subjects participated in the experiment with informed consents. All experiments were approved by the RIKEN Functional MRI Safety and Ethics Committee. These subjects have previously participated in other experiments conducted at the same site. Threedimensional T1-weighted anatomical images and functional retinotopic mapping data are available for these subjects.

Imaging hardware and parameters. All experiments were conducted on a Varian Unity Inova 4 tesla whole-body MRI system (Varian NMR Instruments) equipped with a Magnex head gradient system (Magnex Scientific). A 5 inch transmit/receive quadrature radio-frequency surface coil was used to acquire functional and coregistered anatomical images in the functional experiments. The surface coil was mounted on a Bakelite support frame attached to the patient table. During the experiment, the subject was made to lie supine on the patient table and to rest the back of the head on the surface coil. Head motion was restricted using lateral stabilizer as well as a bite bar. The subject's heartbeat and respiration were 
a

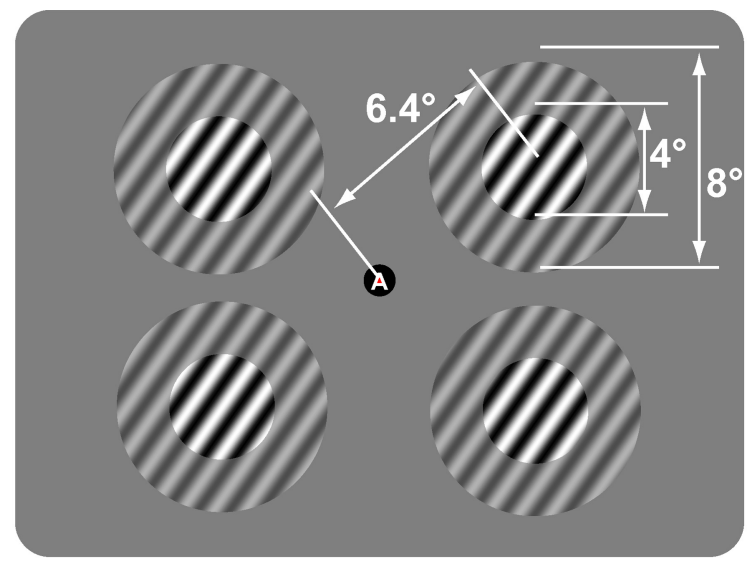

b
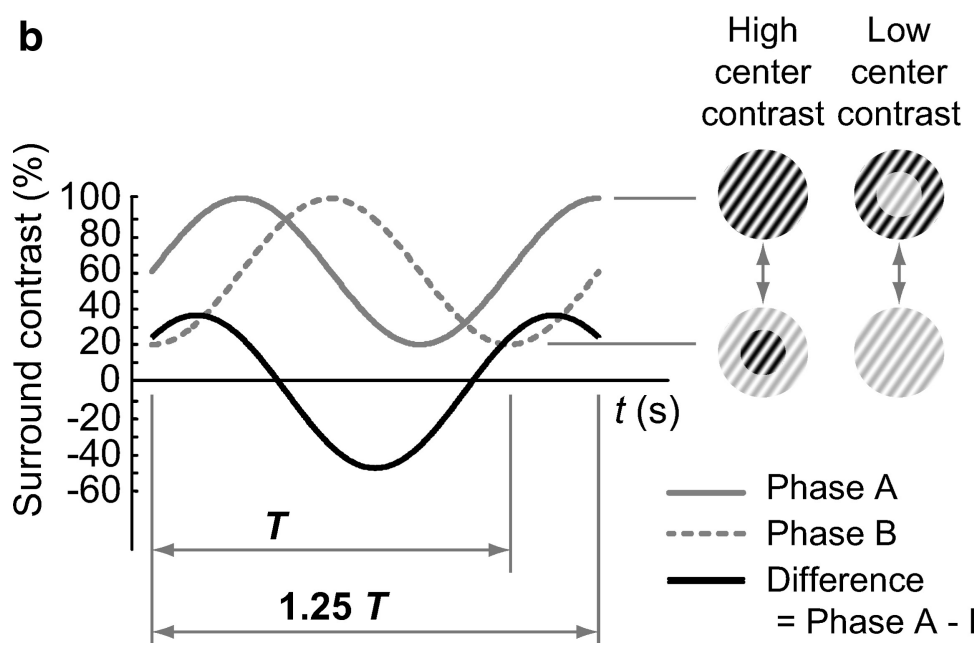

Phase A

Phase B

Difference

$=$ Phase $\mathrm{A}-$ Phase $\mathrm{B}$

Figure 1. The stimulus design. $\boldsymbol{a}$, The stimulus consisted of four sinusoidal grating patches placed in the periphery $\left(6.4^{\circ}\right)$ of the four quadrants. $\boldsymbol{b}$, Left, The temporal structure of the stimulus in the test blocks. The gray solid and dashed curves depict the time courses of the contrast modulation in the surround in two different phase conditions (phase $\mathrm{A}$ : sine $\left[0^{\circ}\right]$; phase B: negative cosine $\left[-90^{\circ}\right]$ ), and the black curve represents the difference of the two phases. The modulation period, $T$, was either $40 \mathrm{~s}$ (subject 2) or $48 \mathrm{~s}$ (subjects 1 and 3). Right, Snapshots illustrating the stimulus appearance when the contrast in the surround changed from high (100\%) to low (20\%). The contrast of the center grating was fixed at $100 \%$ in the HCC condition and $20 \%$ in the LCC condition.

monitored and recorded along with the timing of RF pulses for later corrections of physiological fluctuations. In all experiments, four contiguous slices (in-plane resolution, $1 \times 1 \mathrm{~mm}$; field of view, $12.8 \times 12.8 \mathrm{~cm}$; matrix size, $128 \times 128$; slice thickness, $3 \mathrm{~mm}$ ) were collected. The slice positions were determined in a separate localizer experiment. As the stimulus was peripherally presented, the activated portion was in the calcarine $\mathrm{V} 1$, thus avoiding the confluent foveal representation in the opercular V1. Functional images were acquired using an eight-segment centric-ordered echo-planar imaging (EPI) pulse sequence (volume TR, $2.02 \mathrm{~s}$; TE, $25 \mathrm{~ms}$; average flip angle, $40^{\circ}$ ).

Visual stimulation. The presentation of visual stimuli was controlled by $\mathrm{C}++$ programs and Open GL run on a Windows system. Stimuli were presented on a frosted glass screen placed above the subject's head, using an LCD projector (Avotec, Silent Vision 6011). The projector had an image resolution of $800 \times 600$ pixels and a refresh rate of $60 \mathrm{~Hz}$. The luminosity of the brightest white available on the screen was $74 \mathrm{~cd} / \mathrm{m}^{2}$ and the darkest black was $0.29 \mathrm{~cd} / \mathrm{m}^{2}$. The subject viewed the projected visual image through a mirror mounted in front of the eyes. The wholescreen image subtended $28^{\circ} \times 23^{\circ}$ of the visual angle. The timing of the stimulus onset and offset was synched to the image acquisition by using the trigger signals sent out from the scanner's console to the stimulus presentation PC through a parallel I/O port.

To localize voxels corresponding to the central target stimuli and those to the surrounding annuli used in the main experiment (see below), two additional scans were acquired using a standard block-design paradigm (8 repetitions of $30 \mathrm{~s}$ baseline with a uniform gray and $30 \mathrm{~s}$ test with a flickering checkerboard of $1 \mathrm{cpd}$ and 5 $\mathrm{Hz}$ ) with the same slice positions. In each of the four quadrants, the checkerboard, either the one presented in the center or the one presented in the surround, occupied the same retinotopic region as the test stimulus in the center or the surround used in the main experiment. A central fixation marker was present throughout the scan.

For the test stimulus in the main experiment, we used a block-design paradigm with temporally structured test blocks (Fig. 1a,b). Four sinusoidal grating patches (1 cpd, counter-phase flickering at $5 \mathrm{~Hz}$ ) were simultaneously presented in the periphery $\left(6.4^{\circ}\right.$ away from the fixation) of the four quadrants. Each patch consisted of a circular center region (diameter, $4^{\circ}$ ) and a surrounding annulus (outer diameter, $8^{\circ}$ ) with the same orientation and phase as in the center region. While the orientation of grating was constant $\left(35^{\circ}\right.$ oblique toward right) and identical between the four patches, the spatial phases of the four patches were different, which were pseudorandomly determined at the beginning of each scanning session. There was no gap between the center and surround. The contrast in the center region was kept constant at $20 \%$ and $100 \%$, respectively, for low and high center contrasts. The contrast in the surround was repeatedly modulated between $20 \%$ and $100 \%$ for 1.25 cycles with a period of $40 \mathrm{~s}$ (for subject 2) or $48 \mathrm{~s}$ (for subjects 1 and 3 ) and presented in either sine (phase $\mathrm{A}, 0^{\circ}$ ) or negative cosine (phase $\mathrm{B},-90^{\circ}$ ). The temporal phases of the surround modulation for the four patches in the four visual quadrants were always the same. Each test block was followed by a $20 \mathrm{~s}$ control with a uniform gray background. The A-B-B-A cycle was repeated three times, and in total, each phase condition was tested six times for both high-center-contrast (HCC) and lowcenter-contrast (LCC) conditions.

Throughout the experiment, the subject was instructed to fixate on the central fixation marker $\left(\sim 1^{\circ}\right.$ in size). To help maintain fixation and the level of arousal, the subject performed a letter detection task on the fixation marker. A pseudorandomly ordered temporal sequence of white single characters altering at $5 \mathrm{~Hz}$ was presented on the fixation marker, and subjects reported by button press immediately when they detected a target letter ("S" or "T"). The task performance was checked online and offline, and all the subjects could perform the task almost perfectly.

Data processing and analysis. In all functional experiments, longitudinal magnetization was allowed to reach steady state, using dummy scans, before EPI images were collected. EPI distortions were minimized using a reference volume (without phase encoding) acquired at the beginning of each experiment (Bruder et al., 1992). The first echo in each segment was a navigator echo, which was used to correct intersegment phase and amplitude variations (Kim et al., 1996). After EPI images were reconstructed, cardiac and respiratory fluctuations were removed from time series images using a retrospective estimation and correction method which used pulsation and respiration data recorded during image acquisition (Hu et al., 1995). This correction was applied in $k$-space. Then, twodimensional motion correction was applied in image space on BrainVoyager (Brain Innovation). A high-pass filter was used to suppress baseline signal drifts. This filter was set to have a $3 \mathrm{~dB}$ drop-off cutoff frequency of 0.0002 
cycles/s, but to retain the DC level. No other spatial or temporal smoothing was applied (Forman et al., 1995).

All subsequent data processing and statistical analyses were performed with custom programs written on MATLAB (The MathWorks). For selection of voxels of interest, the voxels activated by the localizing checkerboard were identified by comparing images acquired during stimulation periods and those acquired during control periods on a voxel-by-voxel basis. To account for the hemodynamic delay (typically $4-6 \mathrm{~s}$ ), the first three volumes acquired in each period were excluded. The voxels exhibiting $>2 \%$ of BOLD responses to the center or surround checkerboard were defined as belonging to the center region or the surrounding annulus. We discarded the voxels corresponding to the stimulus boundary between the center and surround. We also eliminated the voxels displaying $>5 \%$ signal changes, which are typically associated with large surface veins (Cheng et al., 2001). We limited our analysis to V1 by discarding the voxels falling outside the calcarine sulcus. To increase the statistical power in the analysis, we pooled all voxels from the four visual quadrants for the center region and surround, respectively. In an additional analysis, we also used the voxels from each of the four quadrants and verified that all the four visual quadrants exhibited similar trends as far as the signal modulation (suppressive or facilitative) is concerned (data not shown).

Several methods were used for analyzing the responses of voxels in the center region and surround. We first calculated the difference of average BOLD responses to phase $\mathrm{A}\left(\right.$ sine, $0^{\circ}$ ) and phase $\mathrm{B}$ (negative cosine, $-90^{\circ}$ ) in either high- or low-center-contrast condition. This approach allowed us to cancel the baseline effect in the BOLD signal due merely to the presence of the stimulus in the center per se as well as the transient onset and offset responses and responses due to neural adaptation, which were common between the two phase conditions. The differential response, in the center region or the surround, was therefore primarily related to the effect of contrast modulation in the surround (Fig. 2a-f). We then fitted the differential responses in the center and surround, respectively, with a sinusoidal function and quantified their amplitudes. To account for the hemodynamic response delay, a shift of three volumes (three data points, or $\sim 6 \mathrm{~s}$ ) was used for the fitting purpose. Since the modulation amplitude in the differential response was amplified by a factor of $\sqrt{2}$ from the two original responses (Fig. $1 b$ ), we divided the resultant amplitude value of the fitted function by $\sqrt{2}$ to reflect the original modulation amplitude (Fig. $2 g$ ), that is, the modulation amplitude used in this study was corrected and given as $r / \sqrt{2}$, where $r$ is the amplitude of the fitted sinusoidal function. Note that the modulation amplitude itself always takes a positive value. To indicate whether the modulation was suppressive or facilitative, modulation amplitudes shown in Figure $2 g$ were presented with the signs to demonstrate whether the modulation was positively or negatively correlated with the contrast modulation in the surround. The polarity of each bar shown in Figure $2 g$ was determined by the phase deviation between the fitted differential response and the difference of the contrast modulation (phase A - phase $\mathrm{B})$ in the visual stimulation. If the phase of fitted differential response was $\theta$, the phase deviation was given by $\Delta \theta=\theta-\pi / 4$ because the differential function of contrast modulation is represented by $\sin (t / T+\pi / 4)+c$ (Fig. $1 b$, black curve). As a result, a positive value was assigned to the modulation amplitude if $-\pi / 2<\Delta \theta \leq \pi / 2$, and a negative value was assigned if $-\pi<\Delta \theta \leq-\pi / 2$ or $\pi / 2<\Delta \theta \leq \pi$.

We further calculated the correlation between responses in the center and surround by presenting the temporally evolved pairs of response trajectories in a two-dimensional plot (Fig. $2 h, i)$. For the same concern about the hemodynamic response delay, both responses in the center and surround were shifted by three volumes in this analysis. Finally, we conducted a voxel by voxel analysis to confirm if the observed suppressive and facilitative effects are preserved at the level of single voxels. For this purpose, we fitted the differential responses with sinusoidal functions for individual voxels in the center and surround, respectively, and quantified their phase differences from the mean phase of voxels in the surround. This analysis was performed for the high- and low-center-contrast conditions separately (Fig. 3).

\section{Results}

\section{A novel visual stimulation and analysis method}

We used a block-design paradigm with temporally structured test blocks, in which the surround contrast was sinusoidally modulated (see Materials and Methods). The reason for us to take this procedure was to cancel the baseline effect of temporal changes in the BOLD signal due merely to the presence of the center grating per se.

Our stimulation paradigm and analysis method have three main advantages over conventional approaches based on block designs. First, we can efficiently obtain an orderly series of cortical responses to almost the entire regime of stimulus contrasts. Second, the slow sinusoidal modulation of the stimulus contrast extricates us from making any detailed assumption on the form of the hemodynamic response function (HRF). The rationale for this statement is as follows. The temporal evolution of BOLD response is well described by the neural response convolved with the HRF. If the stimulus evokes an approximately sinusoidal modulation in the neural response, it is expected that the resulting BOLD response is also sinusoidally modulated, irrelevant to the form of the HRF (for more details and numerical simulations, see supplemental Fig. 1, available at www.jneurosci.org as supplemental material). The drawback of conventional block-design methods is that it typically contains strong transients in the stimulus, about which an explicit form of the HRF needs to be assumed. This often proves to be problematic as any erroneous assumption will lead to inaccurate estimate of BOLD response. Finally, taking the difference between the phase A and phase B conditions, which differ only in the phase of the surround contrast modulation, is critical for extracting the indirect effect by the contextual surround. The constant presence of the stimulus in the central region evokes a DC-like baseline response, which itself contains complex response features, including the adaptation to prolonged stimulation, overshoot at the beginning of stimulus presentation and undershoot at the end (see supplemental Fig. $1 b, c$, available at www.jneurosci.org as supplemental material). By subtracting the response to one phase condition from that to the other, we can extract an AC-like response change due primarily to the change in the surround contrast (supplemental Fig. 1e, available at www.jneurosci.org as supplemental material).

\section{Dependency of surround effect on contrast of center stimulus} The voxels corresponding to the central stimulus and those corresponding to the surrounding annulus were respectively localized in two separate scans using a conventional block-design paradigm (see Materials and Methods). We only analyzed the voxels in the calcarine $\mathrm{V} 1$ in the present study and found that the BOLD response in the center region was indeed modulated by the change in the contrast of the surrounding annulus. Shown in Figure 2, $a$ and $b$, are the average responses from voxels in the center and surround, respectively, under the HCC (100\%) condition for a representative subject (subject 1). The two gray curves in each panel represent original BOLD responses to the two phase conditions of the surround contrast modulation (solid curve, phase A; dashed curve, phase B), and the black solid curve displays the difference between the responses to the two phase conditions. The differential response in the surround exhibits a temporal structure consistent with the contrast modulation in the surround (compare the black curve in Fig. $2 b$ and that in Fig. $1 b)$, demonstrating the change in its direct response to the contrast modulation. On the contrary, the differential response in the center was negatively modulated following the change in the surround contrast (compare the black curve in Fig. $2 a$ and that in 


\section{High center contrast}

a

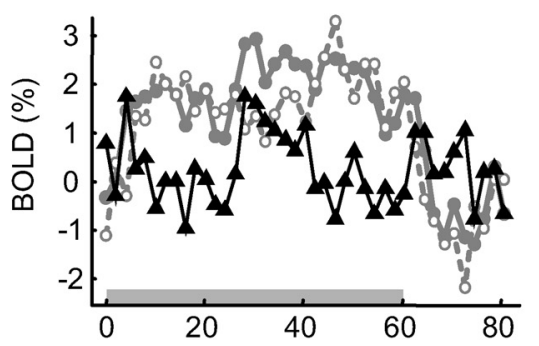

b

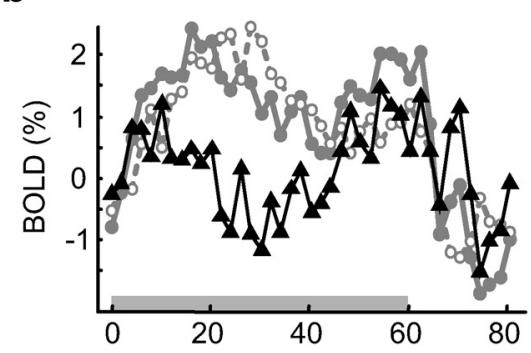

Low center contrast

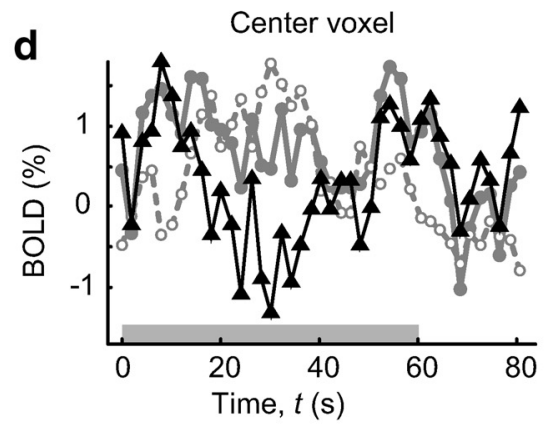

e

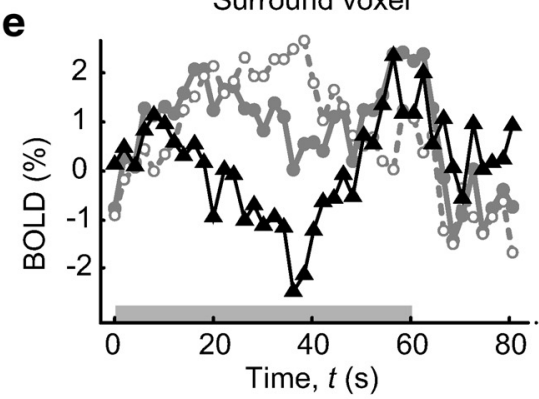

C

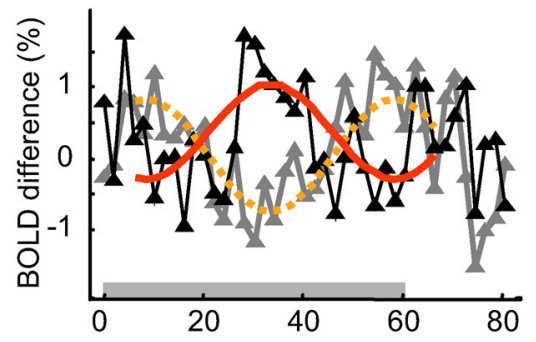

Phase A

$\longrightarrow$ Difference $=$ Phase $A-$ Phase $B$



$\longrightarrow$ Differential response in center - Differential response in surround Stimulus presentation
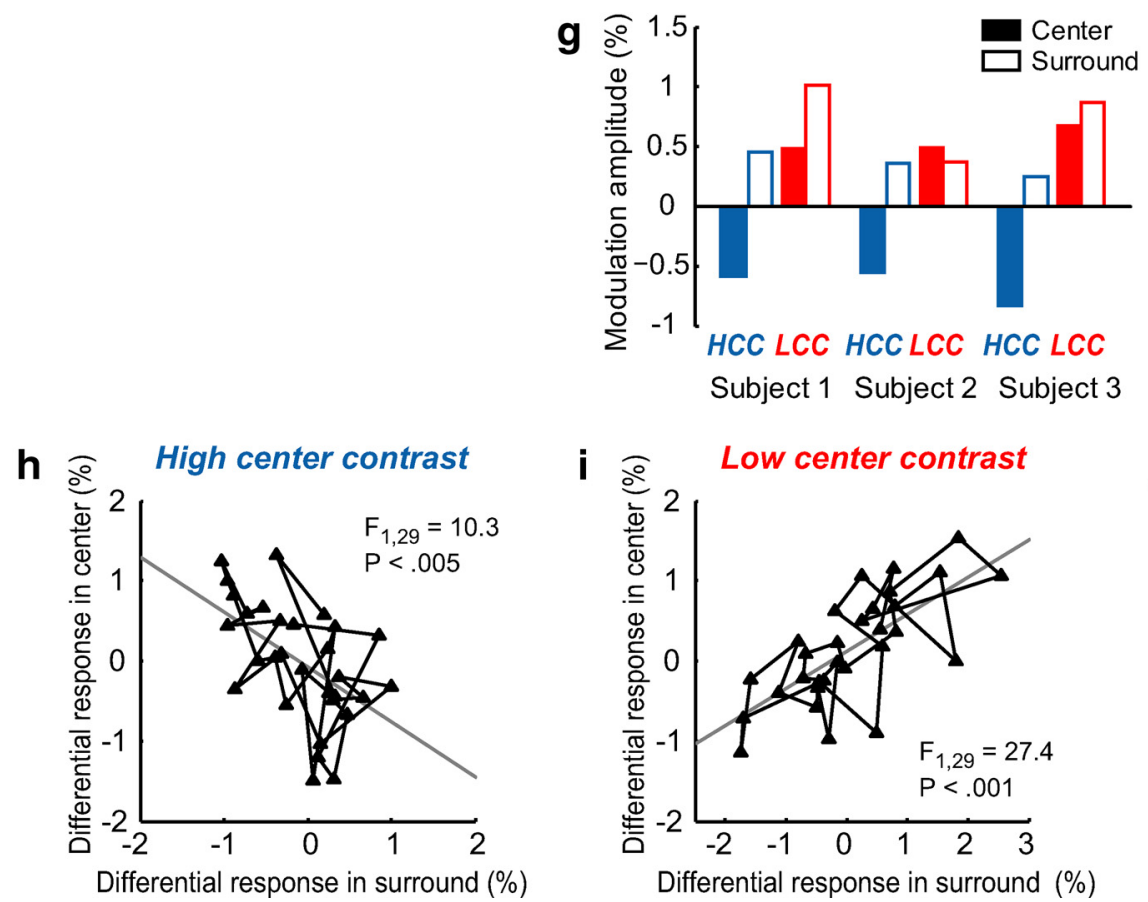

Figure 2. The effects of contextual surround on cortical responses. $\boldsymbol{a}-\boldsymbol{c}$, Voxel- and block-averaged BOLD responses in the HCC condition from a representative subject (subject 1). $\boldsymbol{a}$, The average differential response of voxels in the center region (black curve), which was the difference between the two original responses obtained in phase A (gray solid curve) and phase B (gray dashed curve). $\boldsymbol{b}$, The responses of voxels in the surround. The two black curves in $\boldsymbol{a}$ and $\boldsymbol{b}$ are overlaid in $\boldsymbol{c}$. The red solid and orange dotted curves are the sine wave fits to the differential responses in the center and surround, respectively. $\boldsymbol{d}-\boldsymbol{f}$, Similar results as shown in $\boldsymbol{a}-\boldsymbol{c}$, but for the $\mathrm{LCC}$ condition. $\boldsymbol{g}$, The modulation amplitudes obtained from all three subjects. The blue and red bars are respectively for $\mathrm{HCC}$ and LCC conditions. The filled bars indicate the responses in the center and the open bars show the responses in the surround (excluding the center region). The bar length reflects the average of corrected modulation amplitudes from the sine wave fits and the polarity of a bar indicates whether the modulation was positively or negatively correlated with the contrast modulation in the surround (see Materials and Methods). $\boldsymbol{h}, \boldsymbol{i}$, Correlations between the responses in the center and surround in HCC $(\boldsymbol{h})$ and LCC $(\boldsymbol{i})$ conditions from subject 1. The time points were sampled from the duration of the test blocks, taking the hemodynamic response delay $(6.06 \mathrm{~s}$ or 3 TRs) into consideration. The gray line in each plot is the result of the linear regression. $\boldsymbol{j}$, The modulation ratios, defined by the slope of the linear regression shown in $\boldsymbol{h}$ or $\boldsymbol{i}$, for the three subjects. The blue and red bars represent the modulation ratios in HCC and LCC conditions, respectively. A negative modulation ratio indicates that the contrast modulation in the surround had a suppressive effect on responses of voxels in the center region. 
Fig. $1 b)$. These opposing trends can be clearly captured when the two differential responses are overlaid (Fig. $2 c$ ). The modulated response in the center region is considered to reflect the indirect effect from the surround stimulus (i.e., the contextual effect) because, first, the physical contrast of the central stimulus was kept constant, and second, the baseline response to the central stimulus per se was eliminated by taking the difference of responses to the two phase conditions. This observation verifies previous reports that the effect of the surround on the center was suppressive when the stimulus in the center region was presented with high contrast (Williams et al., 2003; ZengerLandolt and Heeger, 2003).

However, when the same analysis was performed for the data from the LCC (20\%) condition, very different results were observed (Fig. 2d-f). Critically, when the contrast in the center was low, the modulated response in the center region was synchronized to the response in the surround, thus demonstrating a facilitative effect of the surround on the center under such a condition (Fig. 2f). Such a general facilitative effect has not been observed previously in human imaging studies. Since surround stimuli themselves were indifferent between LCC and HCC conditions, this drastic switch in the response in the center region, from suppressive to facilitative (Fig. 2, compare the black curve in $c$ and that in $f$ ), was solely driven by the change in the center contrast from high to low. We also obtained similar results from the remaining two subjects (see supplemental Figs. 2a, $b$, $3 a, b$, available at www.jneurosci.org as supplemental material).

Opposing effects of contextual surround as a general property To quantify the direct and indirect effects of the surround contrast modulation, we performed analyses using both region of interest and single voxel analysis approaches. We first fitted the differential responses in the center and surround (Fig. $2 c, f$, black and gray curves) with a sinusoidal function and analyzed their amplitudes. The results obtained from all three subjects for both HCC and LCC conditions are summarized in Figure $2 g$, where the length of each bar indicates the average of corrected amplitudes of the fitted differential responses from the center region (filled bar) or the surround (open bar), and the sign of each bar indicates whether the modulation was positively or negatively correlated with the contrast modulation in the surround (see Materials and Methods for details). Although there were some quantitative differences between the subjects, for example, the modulation in the center region in subjects 1 was smaller than that in the surround in the LCC condition and the opposite was observed in subject 3 in the HCC condition, it is nevertheless clear that from all the subjects the modulation of responses in the center region was always suppressive in the HCC condition and facilitative in the LCC condition. The results summarized in Figure $2 g$ are also very consistent in showing that the response in the center region was suppressed or facilitated at approximately the same strength within each subject.

We then calculated the correlation between the responses in the center and surround during the stimulus presentation. The results from the same representative subject are plotted in Figure $2, h$ and $i$, for HCC and LCC conditions, respectively, where each point represents a pair of center and surround responses at a given time and is projected to a center vs surround response state space (for plots from the remaining two subjects, see supplemental Figs. $2 c, d, 3 c, d$, available at www.jneurosci.org as supplemental material). Since the surround stimulus was slowly modulated, the responses are temporally evolved as trajectories in the twodimensional plot. If the center response is well explained by the surround response, the trajectory should be confined to a onedimensional subspace and regressed by the surround response, which was indeed the case. The coefficients of the first order $\left(a_{1}\right)$ were significant for both HCC condition (subject 1: $a_{1}=-0.682$, $F_{(1,29)}=10.3, p<0.005 ;$ subject $2: a_{1}=-0.824, F_{(1,23)}=4.32, p<$ 0.05 ; subject 3: $\left.a_{1}=-0.934, F_{(1,29)}=6.78, p<0.05\right)$ and LCC condition (subject 1: $a_{1}=0.464, F_{(1,29)}=27.4, p<0.001$; subject 2: $a_{1}=0.985, F_{(1,23)}=11.2, p<0.005 ;$ subject $3: a_{1}=0.813, F_{(1,29)}=$ $79.0, p<0.001)$. The slope of each linear regression is taken as a "modulation ratio," which indicates how strongly the surrounding visual context affects the responses of voxels in the center relative to its direct effect on the responses in the surround. The results showing the modulation ratios from all the subjects are summarized in Figure $2 j$. We found that the absolute modulation ratios in both HCC and LCC conditions within each subject are in the same order. This additional measure further verifies our observation above that, despite the difference in the modulation amplitudes between the subjects (Fig. $2 g$ ), the strengths of suppression and facilitation, overall, did not differ substantially.

Finally, we analyzed the voxel by voxel variability to confirm that the dependency of the surround modulation on the center contrast is a general property shared among the voxels. Each curve in Figure $3 a$ represents the differential response of a voxel from the HCC condition fitted by a sinusoidal function having 
the same period as the surround contrast modulation. Although there exists some variability, the features depicted in Figure $2 c$ are still recognizable in the single voxel analysis. To better appreciate the difference between the voxels in the center region and those in the surround at a population level, we plotted the difference between the phase of the fitted curve of a single voxel and the mean phase of fitted curves of all voxels in the surround (Fig. $3 b$ ). From the two histograms depicted in Figure $3 b$, it is evident that the voxels in the center and those in the surround cluster separately, with the mean phases of the two populations differing $\sim 180^{\circ}$. In addition, we confirmed that for both the voxels in the center and those in the surround, their average modulation amplitudes were significantly larger than zero (subject 1: center, $F_{(2,37)}=10.1, p<$ 0.001 , surround, $F_{(2,39)}=23.1, p<0.001$; subject 2: center, $F_{(2,25)}=$ 28.0, $p<0.001$, surround, $F_{(2,158)}=63.8, p<0.001$; subject 3: center, $F_{(2,37)}=72.0, p<0.001$, surround, $F_{(2,161)}=117, p<$ $0.001)$. On the other hand, as depicted in Figure $2 f$, when the center contrast was low, the center and surround populations are almost inseparable (Fig. $3 c, d$ ). The average modulation amplitudes of both clusters in the LCC condition were also significantly larger than zero (subject 1: center, $F_{(2,37)}=14.6, p<0.001$, surround, $F_{(2,39)}=21.8, p<0.001$; subject 2: center, $F_{(2,25)}=$ 17.4, $p<0.001$, surround, $F_{(2,158)}=90.5, p<0.001$; subject 3 : center, $F_{(2,37)}=13.1, p<0.001$, surround, $F_{(2,161)}=152, p<$ $0.001)$. We also confirmed that the phase difference of the responses of voxels in the center region changed significantly from the HCC condition to the LCC condition (pairwise test on circular mean; all subjects: $p<0.001$ ). Note that the voxel properties (e.g., center/surround or suppressed/facilitated) are converted into the temporal structures, or "phase," of the BOLD responses by applying the slow sine-wave stimulus modulation, as in the phase encoding technique in the traveling wave method used for exploring cortical retinotopic representation (Wandell et al., 2007). In our case, notably, not only the retinotopy but also the quality of modulation (suppressed or facilitated) in the individual voxel responses are encoded by the response phases. Because the noise components that are not directly relevant to the surround contrast modulation should not contain any periodical components of the modulation frequency, we conclude that the response phase of each center voxel reflects the property of contextual effects.

\section{Discussion}

Imaging studies in human visual cortex are a crucial step toward bridging neurophysiological and perceptual findings in relation to contextual modulation. Several lines of human psychophysical studies on surround effects are suggestive of target contrast dependency, which is consistent with neurophysiological studies in animals. The surround grating generally suppresses the perceived contrast of the suprathreshold contrast target (Ejima and Takahashi, 1985; Xing and Heeger, 2001), whereas with a low-contrast target, the additional collinear grating presented in the surround enhances the subject's ability to detect the presence of the target (Polat and Sagi, 1993). However, the former two studies used a contrast-matching paradigm, while the latter study used a targetdetection paradigm. The difference in the measurement method makes it obscure whether different surround effects observed in these previous studies reflect purely the change in neural representation in early visual cortex or they may also involve additional factors such as decoding strategies in subsequent processing stages related to particular perceptual tasks. Our study, using a novel stimulation and analysis method, enabled us to extract efficiently the modulatory component in cortical responses. Our results demonstrated unambiguously that the BOLD signal in voxels of V1 corresponding to the representation of target is suppressed (or facilitated) for high (or low) contrast target by the collinear surround, thus providing a clear neurophysiological evidence of contrast-dependent contextual modulation in the human visual cortex.

The effects that we observed in the BOLD signal are in general consistent with the results reported in animal studies using single-unit recording (Polat et al., 1998; Sceniak et al., 1999) and intrinsic optical imaging (Toth et al., 1996). It should be noted, however, that our present fMRI study differs from single-unit recording studies in several aspects. First, the BOLD response obtained in a single voxel in our imaging study represents the property of a cell population rather than a single neuron. Therefore, the appropriate interpretation of our results may be that the adaptive center-surround interaction is observed as a trend in a very large neuronal population in early visual cortex. Second, the stimulus size used in our study is much larger than those typically used in single-unit recording studies. Thus, the contrast dependency of the surround modulation revealed in our study can be viewed as a property across a fairly wide visual field in comparison to the receptive fields of single units. Presumably, such a long-range center-surround interaction contains not only influences from bottom-up (Sadakane et al., 2006) and/or horizontal (Somers et al., 1998; Nauhaus et al., 2009) connections but also top-down feedback effects from higher visual areas (Angelucci et al., 2002; Bair et al., 2003).

\section{References}

Angelucci A, Levitt JB, Walton EJS, Hupe JM, Bullier J, Lund JS (2002) Circuits for local and global signal integration in primary visual cortex. J Neurosci 22:8633-8646.

Bair W, Cavanaugh JR, Movshon JA (2003) Time course and time-distance relationships for surround suppression in macaque V1 neurons. J Neurosci 23:7690-7701.

Blakemore C, Tobin EA (1972) A lateral inhibition between orientation detectors in the cat's visual cortex. Exp Brain Res 15:439-440.

Bruder H, Fischer H, Reinfelder HE, Schmitt F (1992) Image reconstruction for echo planar imaging with nonequidistant k-space sampling. Magn Reson Med 23:311-323.

Cheng K, Waggoner RA, Tanaka K (2001) Human ocular dominance columns as revealed by high-field functional magnetic resonance imaging. Neuron 32:359-374.

Ejima Y, Takahashi S (1985) Apparent contrast of a sinusoidal grating in the simultaneous presence of peripheral gratings. Vision Res 25:1223-1232.

Forman SD, Cohen JD, Fitzgerald M, Eddy WF, Mintun MA, Noll DC (1995) Improved assessment of significant activation in functional magnetic resonance imaging (fMRI): use of a cluster-size threshold. Magn Reson Med 33:636-647.

Hu X, Le TH, Parrish T, Erhard P (1995) Retrospective estimation and correction of physiological fluctuation in functional MRI. Magn Reson Med 34:201-212.

Kapadia MK, Ito M, Gilbert CD, Westheimer G (1995) Improvement in visual sensitivity by changes in local context: parallel studies in human observers and in V1 of alert monkeys. Neuron 15:843-856.

Kasamatsu T, Polat U, Pettet MW, Norcia AM (2001) Colinear facilitation promotes reliability of single-cell responses in cat striate cortex. Exp Brain Res 138:163-172.

Kim SG, Hu X, Adriany G, Uğurbil K (1996) Fast interleaved echo-planar imaging with navigator: high resolution anatomic and functional images at 5 tesla. Magn Reson Med 35:895-902.

Knierim JJ, van Essen DC (1992) Neuronal responses to static texture patterns in area V1 of the alert macaque monkey. J Neurophysiol 67:961-980.

Li CY, Li W (1994) Extensive integration field beyond the classical receptive field of cat's striate cortical neurons-classification and tuning properties. Vision Res 34:2337-2355.

Nauhaus I, Busse L, Carandini M, Ringach DL (2009) Stimulus contrast 
modulates functional connectivity in visual cortex. Nat Neurosci $12: 70-76$.

Nelson JI, Frost BJ (1985) Intracortical facilitation among co-oriented, coaxially aligned simple cells in cat striate cortex. Exp Brain Res 61:54-61.

Ohtani Y, Okamura S, Yoshida Y, Toyama K, Ejima Y (2002) Magnetic responses of human visual cortex to illusory contours. Vision Res 42:1825-1835.

Polat U, Norcia AM (1996) Neurophysiological evidence for contrast dependent long-range facilitation and suppression in the human visual cortex. Vision Res 36:2099-2109.

Polat U, Sagi D (1993) Lateral interactions between spatial channels: suppression and facilitation revealed by lateral masking experiments. Vision Res 33:993-999.

Polat U, Mizobe K, Pettet MW, Kasamatsu T, Norcia AM (1998) Collinear stimuli regulate visual responses depending on cell's contrast threshold. Nature 391:580-584.

Sadakane O, Ozeki H, Naito T, Akasaki T, Kasamatsu T, Sato H (2006) Contrast-dependent, contextual response modulation in primary visual cortex and lateral geniculate nucleus of the cat. Eur J Neurosci 23:1633-1642.

Sceniak MP, Ringach DL, Hawken MJ, Shapley R (1999) Contrast's effect on spatial summation by macaque V1 neurons. Nat Neurosci 2:733-739.
Sillito AM, Grieve KL, Jones HE, Cudeiro J, Davis J (1995) Visual cortical mechanisms detecting focal orientation discontinuities. Visual cortical mechanisms detecting focal orientation discontinuities. Nature 378:492-496.

Somers DC, Todorov EV, Siapas AG, Toth LJ, Kim DS, Sur M (1998) A local circuit approach to understanding integration of long-range inputs in primary visual cortex. Cereb Cortex 8:204-217.

Toth LJ, Rao SC, Kim DS, Somers D, Sur M (1996) Subthreshold facilitation and suppression in primary visual cortex revealed by intrinsic signal imaging. Proc Natl Acad Sci U S A 93:9869-9874.

Wandell BA, Dumoulin SO, Brewer AA (2007) Visual field maps in human cortex. Neuron 56:366-383.

Williams AL, Singh KD, Smith AT (2003) Surround modulation measured with functional MRI in the human visual cortex. J Neurophysiol 89: 525-533.

Xing J, Heeger DJ (2001) Measurement and modeling of center-surround suppression and enhancement. Vision Res 41:571-583.

Zenger-Landolt B, Heeger DJ (2003) Response suppression in V1 agrees with psychophysics of surround masking. J Neurosci 23:6884-6893.

Zipser K, Lamme VA, Schiller PH (1996) Contextual modulation in primary visual cortex. J Neurosci 16:7376-7389. 